\section{RMD Open}

Rheumatic \& Musculoskeletal Diseases

\begin{tabular}{c|l}
$\substack{\text { European } \\
\text { Reference } \\
\text { Network }}$ & $\begin{array}{l}\text { Connective Tissue and Musculoskeletd } \\
\text { Diseases (ERN RecONNET) }\end{array}$
\end{tabular}

\title{
Systemic lupus erythematosus: state of the art on clinical practice guidelines
}

Farah Tamirou, ${ }^{1}$ Laurent Arnaud, ${ }^{2}$ Rosaria Talarico, ${ }^{3}$ Carlo Alberto Scirè, ${ }^{4}$ Tobias Alexander, ${ }^{5}$ Zahir Amoura, ${ }^{6}$ Tadej Avcin, ${ }^{7}$ Alessandra Bortoluzzi, ${ }^{4}$ Ricard Cervera, ${ }^{8}$ Fabrizio Conti, ${ }^{9}$ Alain Cornet, ${ }^{10}$ Hervé Devilliers, ${ }^{11}$ Andrea Doria, ${ }^{12}$ Micol Frassi, ${ }^{13}$ Micaela Fredi, ${ }^{13}$ Marcello Govoni, ${ }^{4}$ Frederic Houssiau, ${ }^{1}$ Ana Lladò, ${ }^{14}$ Carla Macieira, ${ }^{15}$ Thierry Martin, ${ }^{16}$ Laura Massaro, ${ }^{9}$ Maria Francisca Moraes-Fontes, ${ }^{14}$ Cristina Pamfil, ${ }^{16}$ Sabrina Paolino, ${ }^{17}$ Chiara Tani, ${ }^{18}$ Sander W Tas, ${ }^{19,20}$ Maria Tektonidou, ${ }^{21}$ Angela Tincani, ${ }^{13}$ Ronald F Van Vollenhoven, ${ }^{22}$ Stefano Bombardieri, ${ }^{23}$ Gerd Burmester, ${ }^{5}$ João Eurico Fonseca, ${ }^{15}$ Ilaria Galetti, ${ }^{24}$ Eric Hachulla, ${ }^{25}$ Ulf Mueller-Ladner, ${ }^{26}$ Matthias Schneider, ${ }^{27}$ Vanessa Smith, ${ }^{28}$ Maurizio Cutolo, ${ }^{17}$ Marta Mosca, ${ }^{18,29}$ Nathalie Costedoat-Chalumeau ${ }^{30,31,32}$

\section{ABSTRACT}

Systemic lupus erythematosus (SLE) is the paradigm of systemic autoimmune diseases characterised by a wide spectrum of clinical manifestations with an unpredictable relapsing-remitting course. The aim of the present work was to identify current available clinical practice guidelines (CPGs) for SLE, to provide their review and to identify physicians' and patients' unmet needs. Twenty-three original guidelines published between 2004 and 2017 were identified. Many aspects of disease management are covered, including global disease management, lupus nephritis and neuropsychiatric involvement, management of pregnancies, vaccinations and comorbidities monitoring. Unmet needs relate with disease management of some clinical manifestations and adherence to treatment. Many patient's unmet needs have been identified starting with faster diagnosis, need for more therapeutic options, guidelines on lifestyle issues, attention to quality of life and adequate education.

\section{INTRODUCTION}

Systemic lupus erythematosus (SLE) is the paradigm of systemic autoimmune diseases characterised by a wide spectrum of clinical manifestations with an unpredictable relapsing-remitting course. While paediatric cases are described, SLE typically affects women between 16 years and 55 years. It is a heterogeneous condition, which may involve almost all organs and tissues. Some of the most common clinical features are mucocutaneous lesions, arthritis, renal involvement, haematological disorders, serositis and fever. Forty per cent to $70 \%$ of SLE patients suffer from lupus nephritis (LN) whose dominant feature is proteinuria usually associated with

\section{Key messages}

What is already known about this subject?

- Systemic lupus erythematosus (SLE) is the paradigm of systemic autoimmune diseases characterised by a wide spectrum of clinical manifestations with an unpredictable relapsing-remitting course.

- A good number of Clinical Practice Guidelines are currently available on SLE.

What does this study add?

- This review represents a state of the art on existing Clinical Practice Guidelines and unmet needs in SLE.

How might this impact on clinical practice?

- In the framework of the European Reference Network on Rare and Complex Connective Tissue and Musculoskeletal Diseases (ERN ReCONNET), clinicians and patients will collaborate closely to address the unmet needs identified with the aim of harmonising the careprovided to SLE patients.

urinary sediment abnormalities. Between $10 \%$ and $20 \%$ of patients with $\mathrm{LN}$ will develop chronic renal failure. Neuropsychiatric manifestations can also occur such as severe headache, seizure disorder, psychosis, acute confusional state and cognitive dysfunction. A higher rate of mortality and morbidity is associated with renal and neuropsychiatric involvements. The serological picture of SLE is characterised by the positivity of many autoantibodies among which the most specific are anti-dsDNA and anti-Sm. The presence of antiphospholipid antibodies is associated with a worse prognosis. During the course of SLE, patients may accrue both disease-related 
and treatment-related damage. Although better use of available therapies has greatly improved outcome, SLE is still associated with a significant morbidity. In view of the large amount of specialists potentially involved in the daily care of SLE patients, as well as the various therapeutic approaches, it is important to establish a commonly shared treatment strategy. Clinical practice guidelines (CPGs) are systematically developed statements to assist practitioner and patient decisions about appropriate healthcare for specific clinical circumstances. ${ }^{1}$ CPGs have been proposed for SLE, but they are sparse and not homogeneous. This manuscript intended is aimed at identifying current available CPGs for SLE and physician's and patients' unmet needs.

\section{METHODS}

ERN Rare CONnective tissue and musculoskeletal diseases NETwork (ReCONNET) SLE core set network

ERN ReCONNET is a European Reference Network funded by the European Union's Health Program to promote better and safer healthcare, define proper organisational assessment and identify standard and cost-effective pathways for the management of Rare and Complex Connective Tissue Diseases. The network includes rheumatologists (adult and paediatric), internists and immunologists from 26 selected centres in eight different countries across Europe.

Within the ERN ReCONNET, the SLE core set network is composed of the members of the network involved in SLE, of FT and NC-C (the official SLE Disease Coordinators, junior and senior) and of two methodologists of the ERN ReCONNET.

The SLE core set network is addressed to focus on the management of all forms of SLE disease manifestations, including rare and complex conditions.

One of the first core set network targets was to identify the currently available CPGs pertaining to SLE, in order to identify potential unmet needs, which should be further focused on. A literature search included all the papers published until July 2017. Analysis was conducted between June 2017 and February 2018. Planning and evaluation of the work was driven by regular interactions between participants of the working group during meetings (European League Against Rheumatism - EULAR congress 2017, American College of Rheumatology -ACR congress 2017, ERN ReCONNET meeting in Pisa, 4-6 of February 2018), web conferences, emails and the ERN Collaborative Platform (https://webgate.ec.europa.eu).

\section{Systematic literature search}

We carried out a systematic search in PubMed and Embase based on controlled terms (MeSH and Emtree) and keywords and on publication type (CPGs), in order to identify existing CPGs on diagnosis, monitoring and treatment, according to the Institute of Medicine 2011 definition: clinical practice guidelines are statements that include recommendations intended to optimise patient care that are informed by a systematic review of evidence and an assessment of the benefits and harms of alternative care options.

The search strategy is: MEDLINE (PubMed): ('lupus erythematosus, systemic' [MeSH Terms] OR ('lupus' [All Fields] AND 'erythematosus' [All Fields] AND 'systemic'[All Fields]) OR 'systemic lupus erythematosus'[All Fields] OR ('systemic'[All Fields] AND 'lupus'[All Fields] AND 'erythematosus' [All Fields])) AND ('Practice Guideline'[Publication Type] OR 'Practice Guidelines As Topic'[MeSH Terms] OR Practice Guideline [Publication Type] OR 'Practice Guideline' [Text Word] OR 'Practice Guidelines'[Text Word] OR 'Guideline' [Publication Type] OR 'Guidelines As Topic' [MeSH Terms] OR Guideline[Publication Type] OR 'Guideline'[Text Word] OR 'Guidelines'[Text Word] OR 'Consensus Development Conference'[Publication Type] OR 'Consensus Development Conferences As Topic' [MeSH Terms] OR 'Consensus' [MeSH Terms] OR 'Consensus'[Text Word] OR 'Recommendation'[Text Word] OR 'Recommendations'[Text Word] OR 'Best Practice'[Text Word] OR 'Best Practices' [Text Word]). Embase: ('lupus erythematosus'/exp OR 'chronic lupus erythematosus' OR 'lupus erythematodes' OR 'lupus erythematosus' OR 'lupus erythematosus' OR 'lupus erythematosus treatment' OR 'lupus syndrome') AND ('practice guideline'/exp OR 'practice guideline' OR 'practice guidelines'/exp OR 'practice guidelines' OR 'clinical practice guideline'/exp OR 'clinical practice guideline' OR 'clinical practice guidelines' / exp OR 'clinical practice guidelines' OR 'clinical practice guidelines as topic'/exp OR 'clinical practice guidelines as topic' OR 'guideline' / exp OR 'guideline' OR 'guidelines' / exp OR 'guidelines' OR 'guidelines as topic'/exp OR 'guidelines as topic' OR 'consensus development'/exp OR 'consensus development' OR 'consensus development conference'/exp OR 'consensus development conference' OR 'consensus development conferences' / exp OR 'consensus development conferences' OR 'consensus development conferences as topic'/exp OR 'consensus development conferences as topic' OR 'consensus' / exp OR 'consensus' OR 'recommendation' OR 'recommendations') AND [embase]/lim NOT [medline]/lim.

In order to implement the list of guidelines provided by MEDLINE and Embase search, the group also performed a hand search.

\section{Methodology of CPGs identification}

All references included in the final list (systematic search+hand search) identified during the systematic literature search were screened for eligibility by two evaluators, the Disease Coordinators (NC-C and FT) of the ERN ReCONNET for SLE, based on title and abstract assessment. We addressed the following question: does this paper describe CPG? Manuscripts scored as such by at least one of the two evaluators were included in the next step.

The two evaluators then assessed all selected references with the full article in order to confirm that they were 


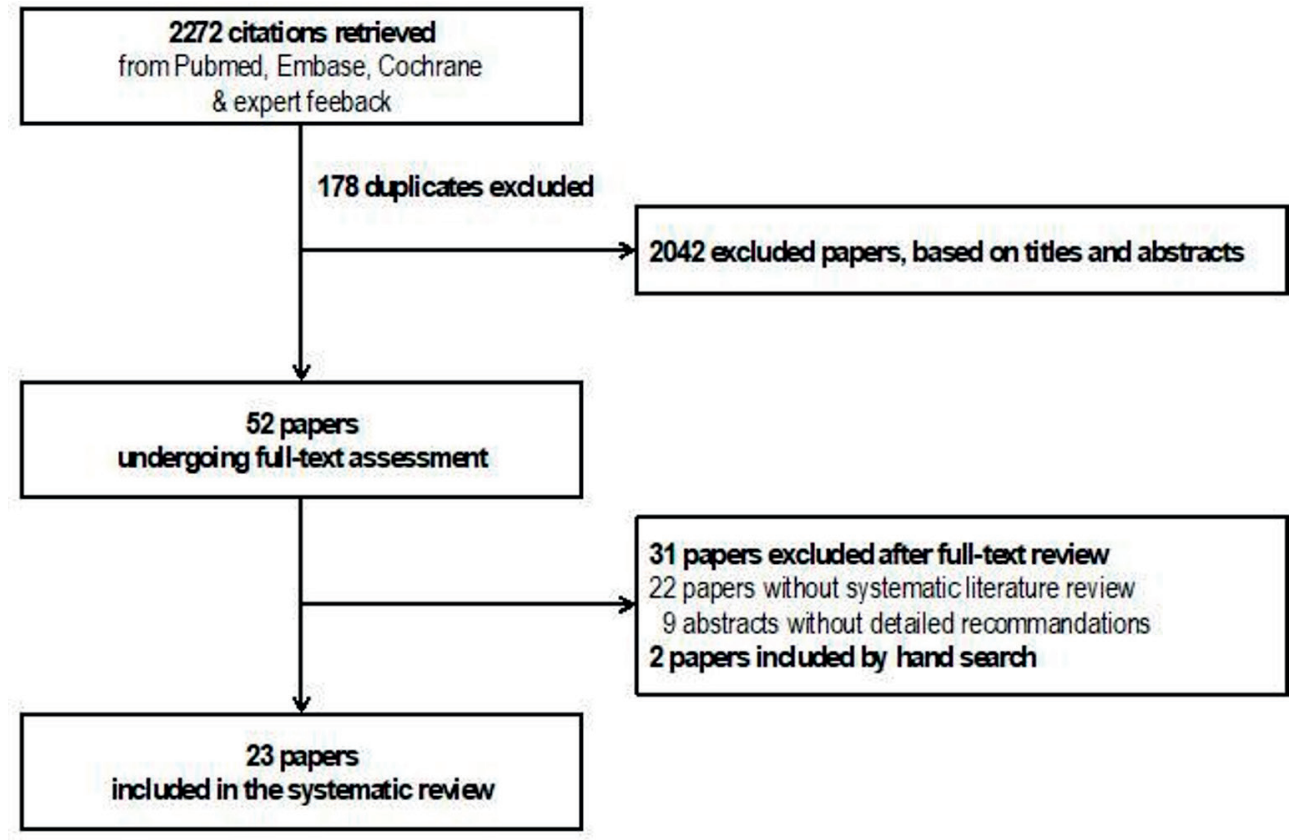

Figure 1 Flowchart constructed from Pubmed, Embase, and national databases.

CPGs. In case of no agreement, a further round of discussion involving a third evaluator (LA) was performed, in order to reach consensus.

A discussion group was set up to confirm inclusion and evaluation of the selected CPGs. The topics covered by each guideline were systematically evaluated by one member of the group (FF) in order to guide the discussion group during the identification of the unmet needs. Physician's unmet needs were then defined by the group, each participant giving his thoughts regarding what is not currently addressed by the current guidelines.

Finally, the patient's unmet needs paragraph intends to highlight the unmet needs of the European lupus community. The content of this paragraph has been realised by the ERN ReCONNET European Patient Advocacy Group that carefully collected the voices and the points of view of the whole European community of the disease they represent by means of meetings and web conferences.

\section{RESULTS}

State of the art on CPGs

Identification of existing CpGs

The systematic literature search yielded a total of 2272 citations. Title and abstract evaluation identified 52 papers suitable for full-text review. After full-text review, 21 original guidelines were identified ${ }^{2-23}$ (figure 1. Of note, Saavedra et al published one guideline, which is divided into two parts with two different references, but this guideline was counted as one in the systematic search. ${ }^{1516}$ Two articles were included by hand search, ${ }^{24}{ }^{25}$ leading to a total of 23 CPGs.

The general characteristics of the 23 CPGs are summarised in table 1. Twenty-one were in English (including one in both English and Portuguese ${ }^{8}$ ) and two in French. Sixteen guidelines had been endorsed/ supported by an official society or organisation: European League Against Rheumatism (EULAR) $(\mathrm{n}=8)$, American College of Rheumatology (ACR) ( $\mathrm{n}=3$ ), Brazilian Society of Rheumatology $(\mathrm{n}=1)$, European Union (SHARE initiative) $(n=2)$, Mexican College of Rheumatology $(n=1)$ and Italian Society of Laboratory Medicine $(n=1)$. The guidelines were published between 2004 and 2017 with only four published before 2010 .

Five CPGs involved patient representatives and one involved a patient panel. Fifteen CPGs were dedicated to SLE, while eight covered a broader spectrum of rheumatic diseases (including SLE). Seventeen targeted all patients (juvenile and adult), four papers specifically targeted juvenile SLE and two female SLE. Five CPGs addressed general management of SLE, five addressed prevention or treatment of infections (three specifically focusing on vaccination), four focused on a specific SLE organ involvement (three on renal disease and one on neuropsychiatric disease) two addressed immunologic laboratory testing, while others focused on pregnancy and family planning $(n=2)$, cardiovascular risk management $(n=2)$, cancer $(n=1)$, orthopaedic perioperative management $(n=1)$ or fatigue $(n=1)$.

\section{UNMET NEEDS}

Clinicians' unmet needs

This review provides an overview of currently available CPGs for SLE. Yet, there are several areas that are not (yet) covered by guidelines.

The following items were considered as correctly covered: (1) global management of SLE, ${ }^{5} 10142022$ including a treatto-target strategy ${ }^{22} ;$ (2) autoantibodies testing ${ }^{4}$; 


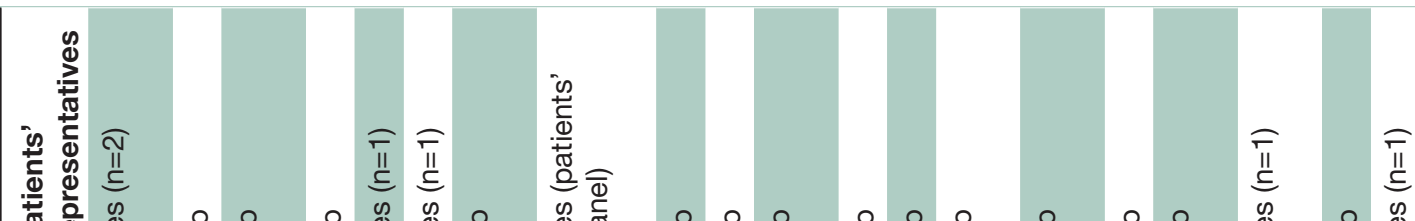

¿

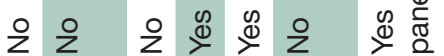

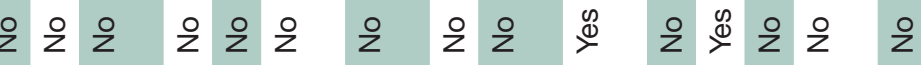

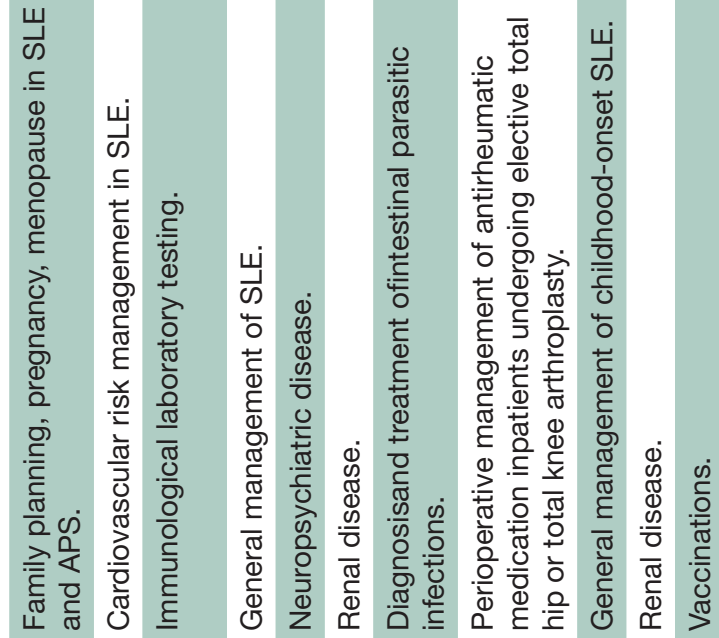

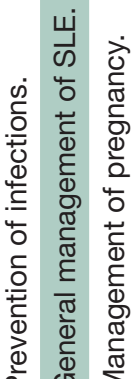

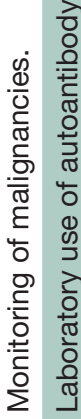

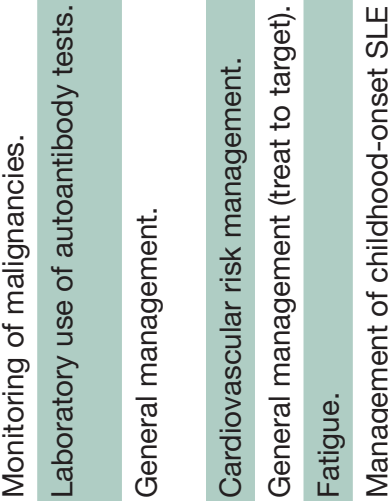

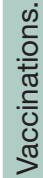

要

흫

๑

o

$\dot{\vec{\omega}}$

害

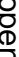

옹

\&
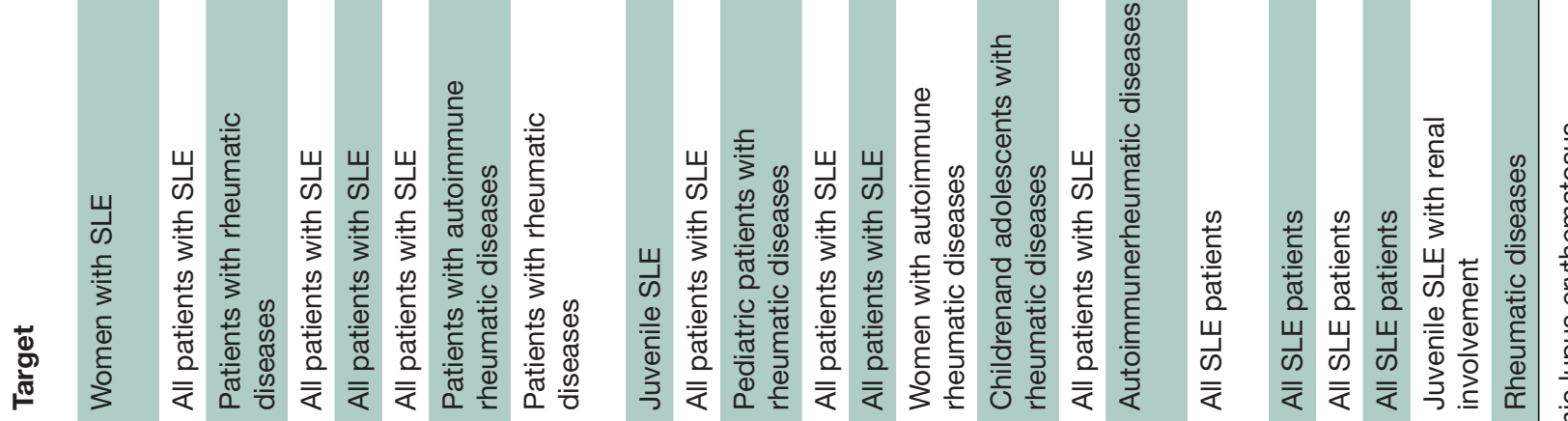

ปั๊
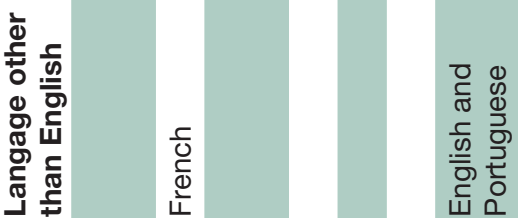

$\frac{\frac{c}{\mathcal{C}}}{\frac{0}{0}}$
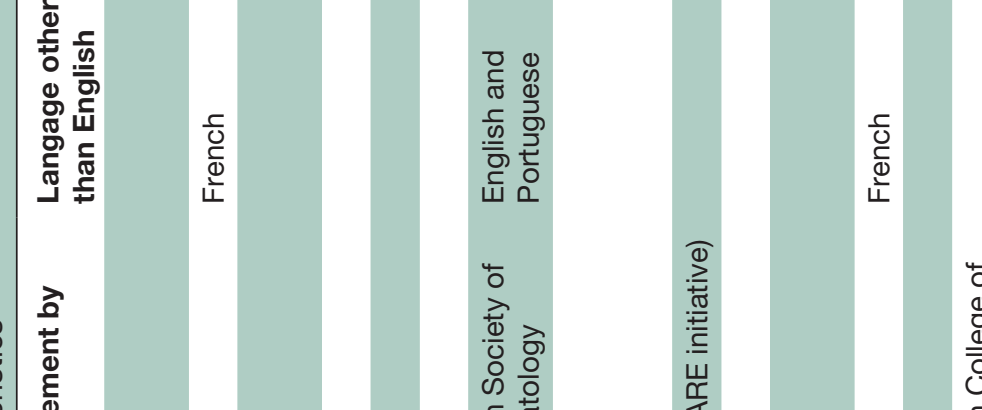

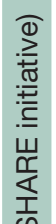

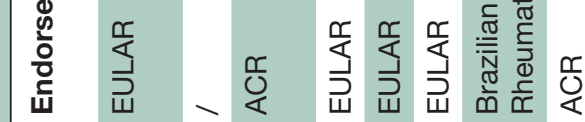
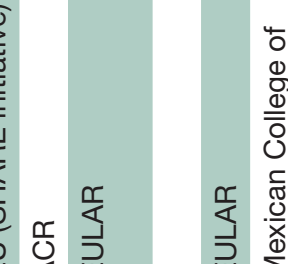

\begin{tabular}{l}
4 \\
0 \\
0 \\
8 \\
\hline 0 \\
\hline 0 \\
0
\end{tabular}

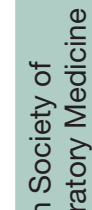

$\frac{0}{\frac{C}{0}}$

눈료

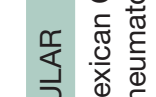

政

管敦

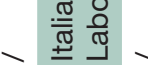


management of fatigue ${ }^{23} ;$ (4) monitoring for malignancies ${ }^{18}$; Screening and management of cardiovascular risk factors ${ }^{3}$ and coronary disease risk monitoring ${ }^{21}$; (5) management (including treatment) of the two most severe manifestations of SLE, namely lupus nephritis ${ }^{71}$ (including in children $^{24}$ ) and neuropsychiatric involvement ${ }^{6}$; (6) prevention of infections ${ }^{13}$ with a focus on intestinal parasitic infections ${ }^{8}$; (7) vaccination in adults, ${ }^{25}$ in paediatric patients ${ }^{12} 17$ and in adolescents $^{17}$; (8) pregnancy planning ${ }^{2} 1516$ and management of menopause ${ }^{2}$; and (9) Perioperative management for hip and knee surgery. ${ }^{9}$

By contrast, several clinician's unmet needs were identified: (1) optimal management of serositis, gastrointestinal involvement, interstitial lung disease, retinal vasculitis, limited cutaneous disease, headaches and/or severe lymphopaenia that are not covered by the current available CPGs. (2) Evaluation and management of non-adherence to treatment is a crucial missing point which is only addressed by one available CPG. ${ }^{10}$ (3) Optimal duration of immunosuppression, which is only partly addressed in some guidelines. (4) Patient's input on CPGs is missing. Only one CPG proposed patient assessment as a recommendation, which consisted in an evaluation of her/his quality of life by using a visual analogue scale. ${ }^{14}(5)$ Except one CPG on LN, ${ }^{11}$ none of the available CPGs addressed the important question of ethnicity and its possible impact on disease severity. (6) No definition of photosensitivity and vasculitis is provided in current CPGs. (7) No mention of non-health related prognostic determinants, such as patients' socioeconomic status.

\section{Patients' unmet needs}

The first unmet need identified by patients deals with delay and uncertainty in diagnosis until confirmation by a specialist. This adds to the psychological burden of the disease, which might be aggravated if treatment is delayed. The need for new treatment options, less reliant on steroids and associated with fewer side effects, is a high priority for patients. They advocate a more holistic disease management, going beyond specific symptoms or an 'organ by organ' management, to include a global treatment plan, coordinated by one physician, in casu a lupus expert, who treat them as a 'full person' and takes care, besides the clinical aspects, of the psychological issues. In our working group meetings, lupus patients defined treatment as "any product or activity aiming at improving quality of life", clearly pinpointing the importance of a holistic approach. Patients are looking for scientifically validated patient focused guidelines on lifestyle issues. Research should be conducted jointly by HCPs and patient organisations to identify behaviours or actions that can help patients take day-to-day ownership of their treatment, understanding what to do, or not to do, based on hard data. Even if remission of SLE disease activity has been achieved, many patients still face pain and fatigue. Understanding the drivers would allow building treatment guidelines for those conditions, which is critical to avoid that people facing these symptoms are pushed prematurely out of the labour market. Finally, while a huge amount of information is available to patients on the web, this information is of very low quality, often counterproductive and anxiety generating. There is a need for high-quality therapeutic patient education and for an efficacious way to fight fake news that spread over the internet, for example, by quality certified information, or diffusion of ERN-endorsed recommendations via social media posts.

\section{CONCLUSIONS}

Here we proposed an overview of the current available CPGs on SLE. Many unmet needs have been identified. Soon after we performed the systematic research, two clinical guidelines have been published. ${ }^{26}{ }^{27}$ Gordon et al published the British guideline on SLE, ${ }^{26}$ which proposed recommendations for some of these unmet needs, such as patient reported outcomes (Short Form (SF)-36 and Lupus QoL indices) and for immunosuppression duration. Pons-Estel et $a t^{27}$ published another guideline with a focus on socioeconomic and ethnic-namely in Latin Americans-aspects. Yet, many areas remain uncovered, and efforts are still needed to improve and standardise our daily practice.

\section{Author affiliations}

${ }^{1}$ Rheumatology Department, Cliniques universitaires Saint-Luc, Université catholique deLouvain, Bruxelles, Belgium

${ }^{2}$ Service de rhumatologie, Hôpitaux Universitaires de Strasbourg, Centre National de Referencedes Maladies Auto-immunes et Systémiques Rares RES0, Strasbourg, France

${ }^{3}$ Rheumatology Unit, AOU Pisana, Pisa, Italy

${ }^{4}$ Section of Rheumatology, Department of Medical Sciences, University of Ferrara, Ferrara, Italy

${ }^{5}$ Department of Rheumatology and Clinical Immunology, Charité - University Medicine Berlin, Berlin, Germany

${ }^{6}$ Department of Internal Medicine, Hospital Pitié-Salpêtrière, Assistance PubliqueHôpitaux de Paris, Paris, France

${ }^{7}$ Department of Allergology, Rheumatology and Clinical Immunology, University Children'sHospital, University Medical Centre Ljubljana, Ljubljana, Slovenia

${ }^{8}$ Department of Autoimmune Diseases, Hospital Clínic, Barcelona, Spain

${ }^{9}$ Rheumatology Unit, Dipartimento di Medicina Interna e Specialità Mediche, Università degliStudi di Roma La Sapienza, Rome, Italy

${ }^{10}$ Lupus Europe, Brussels, Belgium

${ }^{11}$ Department of Internal Medicine and Systemic Diseases, François-Mitterrand Teaching Hospital, University of Bourgogne-Franche-Comté, Dijon, France

${ }^{12}$ Rheumatology Unit, Department of Medicine, A0 Padova and University of Padua, Padua, Italy

${ }^{13}$ Rheumatology and Clinical Immunology Unit, Civil Hospital, Brescia, Italy

${ }^{14}$ Unidade de Doenças Auto-imunes/Medicina 7.2, Hospital de Curry Cabral, Centro Hospitalarde Lisboa Central, Lisbon, Portugal

${ }^{15}$ Rheumatology Department, Hospital de Santa Maria, Centro Hospitalar Lisboa Norte, Lisbon Academic Medical Centre, Lisbon, Portugal

${ }^{16}$ Department of Rheumatology, Emergency County Teaching Hospital, Cluj-Napoca, Romania

${ }^{17}$ Research Laboratory and Academic Division of Clinical Rheumatology, Department of Internal Medicine, IRCCS Polyclinic Hospital San Martino, University of Genoa, Genoa, Italy

${ }^{18}$ Rheumatology Unit, AOU Pisana, Pisa, Italy

${ }^{19}$ Amsterdam UMC, Department of Clinical Immunology \& Rheumatology and Department of Experimental Immunology, Amsterdam Infection and Immunity Institute, Meibergdreef 9, University of Amsterdam, Amsterdam, The Netherlands

${ }^{20}$ Amsterdam Rheumatology \& immunology Center (ARC), Academic Medical Center, Amsterdam, The Netherlands

${ }^{21}$ Joint Rheumatology Academic Program, First Department of Propaedeutic Internal Medicine, School of Medicine, National and Kapodistrian University of Athens, Athens, Greece 
${ }^{22}$ Clinical Immunology \& Rheumatology, Amsterdam Rheumatology \& Immunology Center, Academic Medical Center/University of Amsterdam, Amsterdam, The Netherlands

${ }^{23}$ University of Pisa, Pisa, Italy

${ }^{24}$ FESCA - Federation of European Scleroderma Association, Milan, Italy

${ }^{25}$ Département de Médecine Interne et Immunologie Clinique, Centre de Référence des Maladies Systémiques et Auto-Immunes Rares du Nord-Ouest (CERAINO), LIRIC, INSERM, Univ.Lille, CHU Lille, Lille, France

${ }^{26}$ Department of Rheumatology and Clinical Immunology, Kerckhoff Clinic, Bad Nauheim, Germany

${ }^{27}$ Department of Rheumatology, Universitätsklinikum Düsseldorf, Düsseldorf, Germany

${ }^{28}$ Department of Rheumatology, Department of Internal Medicine, Ghent University Hospital, Ghent University, Ghent, Belgium

${ }^{29}$ Rheumatology Unit, University of Pisa, Pisa, Italy

${ }^{30}$ Assistance Publique-Hôpitaux de Paris (AP-HP), Internal Medicine Department,

Cochin Hospital, Referral center for rare autoimmune and systemic diseases, Paris France

${ }^{31}$ Paris Descartes Sorbonne, Paris Cité University, Paris, France

${ }^{32}$ INSERM U 1153, Center for Epidemiology andStatistics Sorbonne Paris Cité (CRESS), Paris, France

Acknowledgements Thanks to all the members of the Steering Committee of the ERN ReCONNET for the huge commitment during this work. A special thank goes to all the members of the ERN ReCONNET team for providing support during all the phases of the Work Package 3.

Contributors All authors contributed to the manuscript.

Funding This publication was funded by the European Union's Health Programme (2014-2020)

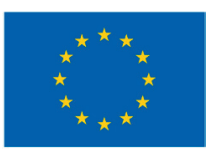

Disclaimer ERN ReCONNET is one of the 24 European Reference Networks (ERNs) approved by the ERN Board of Member States. The ERNs are co-funded by the European Commission. The content of this publication represents the views of the authors only and it is their sole responsibility; it cannot be considered to reflect the views of the European Commission and/or the Consumers, Health, Agriculture and Food Executive Agency (CHAFEA) or any other body of the European Union. The European Commission and the Agency do not accept any responsibility for use that may be made of the information it contains. Competing interests None declared.

Patient consent Not required.

Provenance and peer review Commissioned; externally peer reviewed.

Data sharing statement № additional data are available.

Open access This is an open access article distributed in accordance with the Creative Commons Attribution Non Commercial (CC BY-NC 4.0) license, which permits others to distribute, remix, adapt, build upon this work non-commercially, and license their derivative works on different terms, provided the original work is properly cited, appropriate credit is given, any changes made indicated, and the use is non-commercial. See: http://creativecommons.org/licenses/by-nc/4.0

\section{REFERENCES}

1. Woolf SH, Grol R, Hutchinson A, et al. Clinical guidelines: potential benefits, limitations, and harms of clinical guidelines. BMJ 1999;318:527-30.

2. Andreoli L, Bertsias GK, Agmon-Levin N, et al. EULAR recommendations for women's health and the management of family planning, assisted reproduction, pregnancy and menopause in patients with systemic lupus erythematosus and/or antiphospholipid syndrome. Ann Rheum Dis 2017;76:476-85.

3. Arnaud L, Mathian A, Adoue D, et al. [Screening and management of cardiovascular risk factors in systemic lupus erythematosus: Recommendations for clinical practice based on the literature and expert opinion]. Rev Med Interne 2015;36:372-80.

4. Benito-Garcia E, Schur PH, Lahita R, et al. Guidelines for immunologic laboratory testing in the rheumatic diseases: anti-Sm and anti-RNP antibody tests. Arthritis Rheum 2004;51:1030-44.

5. Bertsias G, loannidis JP, Boletis J, et al. EULAR recommendations for the management of systemic lupus erythematosus. Report of a task force of the eular standing committee for international clinical studies including therapeutics. Ann Rheum Dis 2008;67:195-205.

6. Bertsias GK, loannidis JP, Aringer M, et al. EULAR recommendations for the management of systemic lupus erythematosus with neuropsychiatric manifestations: report of a task force of the EULAR standing committee for clinical affairs. Ann Rheum Dis 2010;69:2074-82.

7. Bertsias GK, Tektonidou M, Amoura Z, et al. Joint European League Against Rheumatism and European Renal AssociationEuropean Dialysis and Transplant Association (EULAR/ERA-EDTA) recommendations for the management of adult and paediatric lupus nephritis. Ann Rheum Dis 2012;71:1771-82.

8. Braz AS, de Andrade CA, da Mota LM, et al. [Recommendations from the Brazilian Society of Rheumatology on the diagnosis and treatment of intestinal parasitic infections in patients with autoimmune rheumatic disorders]. Rev Bras Reumatol 2015;55:368-80.

9. Goodman SM, Springer B, Guyatt G, et al. 2017 American College of Rheumatology/American Association of Hip and Knee Surgeons Guideline for the Perioperative Management of Antirheumatic Medication in Patients With Rheumatic Diseases Undergoing Elective Total Hip or Total Knee Arthroplasty. Arthritis Rheumatol 2017:69:1538-51.

10. Groot N, de Graeff N, Avcin T, et al. European evidence-based recommendations for diagnosis and treatment of childhood-onset systemic lupus erythematosus: the SHARE initiative. Ann Rheum Dis 2017;76:1788-96.

11. Hahn BH, McMahon MA, Wilkinson A, et al. American College of Rheumatology guidelines for screening, treatment, and management of lupus nephritis. Arthritis Care Res 2012;64:797-808.

12. Heijstek MW, Ott de Bruin LM, Bijl M, et al. EULAR recommendations for vaccination in paediatric patients with rheumatic diseases. Ann Rheum Dis 2011;70:1704-12.

13. Mathian A, Arnaud L, Adoue D, et al. [Prevention of infections in adults and adolescents with systemic lupus erythematosus: Guidelines for the clinical practice based on the literature and expert opinion]. Rev Med Interne 2016;37:307-20.

14. Mosca M, Tani C, Aringer M, et al. European League Against Rheumatism recommendations for monitoring patients with systemic lupus erythematosus in clinical practice and in observational studies. Ann Rheum Dis 2010;69:1269-74.

15. Saavedra Salinas MÁ, Barrera Cruz A, Cabral Castañeda AR, et al. Clinical practice guidelines for the management of pregnancy in women with autoimmune rheumatic diseases of the Mexican College of Rheumatology. Part I. Reumatol Clin 2015;11:295-304.

16. Saavedra Salinas MÁ, Barrera Cruz A, Cabral Castañeda AR, et al. Clinical practice guidelines for the management of pregnancy in women with autoimmune rheumatic diseases of the Mexican College of Rheumatology. Part II. Reumatol Clin 2015;11:305-15.

17. Silva $\mathrm{C}$, Terreri M, Barbosa C. Immunization consensus for children and adolescents with rheumatic diseases. Bras J Rheumatol 2009;49:562-89.

18. Tessier-Cloutier B, Clarke AE, Pineau CA, et al. What investigations are needed to optimally monitor for malignancies in SLE? Lupus 2015;24:781-7.

19. Tozzoli R, Bizzaro N, Tonutti E, et al. Guidelines for the laboratory use of autoantibody tests in the diagnosis and monitoring of autoimmune rheumatic diseases. Am J Clin Pathol 2002;117:316-24.

20. Trujillo-Martín MM, Rúa-Figueroa Fernández de Larrinoa I, RuízIrastorza G, et al. [Clinical practice guidelines for systemic lupus erythematosus: Recommendations for general clinical management] Med Clin 2016;146:413.e1-413.e14

21. Tselios K, Sheane BJ, Gladman DD, et al. Optimal monitoring for coronary heart disease risk in patients with systemic lupus erythematosus: a systematic review. J Rheumatol 2016;43:54-65.

22. van Vollenhoven RF, Mosca M, Bertsias G, et al. Treat-to-target in systemic lupus erythematosus: recommendations from an internationa task force. Ann Rheum Dis 2014;73:958-67.

23. Yuen HK, Cunningham MA. Optimal management of fatigue in patients with systemic lupus erythematosus: a systematic review. Ther Clin Risk Manag 2014;10:775-86.

24. Groot N, de Graeff N, Marks SD, et al. European evidence-based recommendations for the diagnosis and treatment of childhood-onset lupus nephritis: the SHARE initiative. Ann Rheum Dis 2017;76:1965-73.

25. van Assen S, Agmon-Levin N, Elkayam O, et al. EULAR recommendations for vaccination in adult patients with autoimmune inflammatory rheumatic diseases. Ann Rheum Dis 2011;70:414-22.

26. Gordon C, Amissah-Arthur MB, Gayed M, et al. The British Society for Rheumatology guideline for the management of systemic lupus erythematosus in adults. Rheumatology 2018;57:e1-e45.

27. Pons-Estel BA, Bonfa E, Soriano ER. First Latin American clinical practice guidelines for the treatment of systemic lupus erythematosus: Latin American Group for the Study of Lupus (GLADEL, Grupo Latino Americano de Estudio del Lupus)-PanAmerican League of Associations of Rheumatology (PANLAR). Ann Rheum Dis 2018. 